\title{
Simpler completeness proofs for modal logics with intersection
}

\author{
Yì N. Wáng ${ }^{1}$ and Thomas Ågotnes ${ }^{2,3}$ \\ 1 Zhejiang University, Hangzhou, China \\ ynw@xixilogic.org \\ 2 University of Bergen, Bergen, Norway \\ 3 Southwest University, Chongqing, China \\ Thomas.Agotnes@infomedia.uib.no
}

\begin{abstract}
There has been a significant interest in extending various modal logics with intersection, the most prominent examples being epistemic and doxastic logics with distributed knowledge. Completeness proofs for such logics tend to be complicated, in particular on model classes such as S5 like in standard epistemic logic, mainly due to the undefinability of intersection of modalities in standard modal logics. A standard proof method for the S5 case was outlined in 8 and later explicated in more detail in [13, using an "unraveling-folding method" case to achieve a treelike model to deal with the problem of undefinability. This method, however, is not easily adapted to other logics, due to the level of detail and reliance on S5. In this paper we propose a simpler proof technique by building a treelike canonical model directly, which avoids the complications in the processes of unraveling and folding. We demonstrate the technique by showing completeness of the normal modal logics $\mathrm{K}, \mathrm{D}, \mathrm{T}$, B, S4 and S5 extended with intersection modalities. Furthermore, these treelike canonical models are compatible with Fischer-Ladner-style closures, and we combine the methods to show the completeness of the mentioned logics further extended with transitive closure of union modalities known from PDL or epistemic logic. Some of these completeness results are new.
\end{abstract}

Keywords: modal logic $\cdot$ intersection modality $\cdot$ union modality $\cdot$ completeness $\cdot$ epistemic logic $\cdot$ distributed knowledge.

\section{Introduction}

Intersection plays a role in several areas of modal logic, including epistemic logics with distributed knowledge [117], propositional dynamic logic with intersection of programs [9, description logics with concept intersection [2]3, and coalition logic [1]. It is well-known that intersection is not modally definable and that standard logics with intersection are not canonical (cf., e.g., [10]).

A method for proving completeness was introduced by 8/107/11 for various (static) epistemic logics with distributed knowledge, and later explicated and extended in 1314112 as the unraveling-folding method which is applicable to 
various static or dynamic epistemic S5 logics with distributed knowledge with or without common knowledge.

Let us take a closer look at this technique for epistemic logic with distributed knowledge (S5D). It is known that the canonical S5 model built in the standard way is not a model for the classical axiomatization for this logic. This is because the accessibility relation $R_{G}$ (where $G$ is a set) that is (implicitly) used to interpret the interesection (distributed knowledge) modality is not necessarily the intersection of individual accessibility relations $R_{a}(a \in G)$. In the canonical S5 model we can ensure that $R_{G} \subseteq \bigcap_{a \in G} R_{a}$, but not that $R_{G} \supseteq \bigcap_{a \in G} R_{a}$.

The unraveling-folding method is carried out in the following way. A premodel is a standard S5 model where $R_{G}$ is treated as a primitive relation for each group $G$. A pseudo model is a pre-model satisfying the following two constraints

1. $R_{\{a\}}=R_{a}$ for every agent $a$, and

2. $R_{G} \subseteq \bigcap_{a \in G} R_{a}$ for every agent $a$ and group $G$

An S5D model is then a pseudo model that satisfies also a third constraint:

\section{3. $R_{G} \supseteq \bigcap_{a \in G} R_{a}$ for every agent $a$ and group $G$}

A canonical pseudo model can be truth-preservingly translated to a treelike premodel using an unraveling technique, and then folded to an S5D model while also preserving the truth of all formulas (for details of the two processes see [13]). Completeness is achieved by first building a canonical pseudo model for a given consistent set $\Phi$ of formulas, and then having it translated to an S5D model for $\Phi$ using the unraveling-folding method.

There are many subtleties not mentioned in this simplified overview, which in particular makes the method cumbersome to adapt to extensions of basic epistemic logic or to non-S5 based logics.

In this paper we demonstrate a simpler way to prove completeness for modal logics with intersection. Since we know that a treelike model typically works for such logics, the idea is to build a treelike model directly for a given consistent set of formulas. We call such a model a standard model. This eliminates having to deal with the details of the unraveling and folding processes, and dramatically simplifies the proofs.

We illustrate the technique by building the standard model for each of the modal logics, K, D, T, B, S4 and S5, extended with intersection. We furthermore demonstrate that the method is useful by showing that it is compatible with finitary methods based on Fischer-Ladner-style closures, and introduce finitary standard models for the mentioned logics further extended with the transitive closure of the union, using in, e.g., PDL and epistemic logic, as well. Some of these completeness results have been stated in the literature before, often without proof, some of them not. For example, to the best of our knowledge, no completeness results have been reported for D or B extended with intersection, and even less can be found for logics with both intersection and the transitive closure of union. 
The rest of the paper is structured as follows. In the next section we introduce basic definitions and conventions. We study some modal logics with (only) intersection in Section 3, introduce an axiomatization for each of them and show its completeness, and then study the logics extended further with transitive closure of union in Section 4. We conclude in Section 5 .

\section{Preliminaries}

In this paper we study modal logics over multi-modal languages with countably many standard unary modal operators: $\square_{0}, \square_{1}, \square_{2}$, etc. On top of these we focus on two types of modal operators, each indexed by a finite nonempty set $I$ of natural numbers:

- Intersection modalities, denoted $\cap_{I}$;

- Union ${ }^{+}$modalities, denoted $\uplus_{I}$.

We mention some applications of these modalities below.

The languages are parameterized by a countably infinite set PROP of propositional variables, and an at-most countable set $\mathcal{I}$ of primitive types. A finite non-empty subset $I \subseteq \mathcal{I}$ is called an index. We are interested in the following languages.

\section{Definition 1 (languages).}

$$
\begin{aligned}
& (\mathcal{L}) \quad \varphi::=p|\neg \varphi|(\varphi \rightarrow \varphi) \mid \square_{i} \varphi \\
& \left(\mathcal{L}^{\cap}\right) \quad \varphi::=p|\neg \varphi|(\varphi \rightarrow \varphi)\left|\square_{i} \varphi\right| \cap_{I} \varphi \\
& \left(\mathcal{L}^{\cap \uplus}\right) \varphi::=p|\neg \varphi|(\varphi \rightarrow \varphi)\left|\square_{i} \varphi\right| \cap_{I} \varphi \mid \uplus_{I} \varphi
\end{aligned}
$$

where $p \in \mathrm{PROP}, i \in \mathcal{I}$ and $I$ an index. Other Boolean connectives are defined as usual.

A Kripke model $M$ (over PROP and $\mathcal{I}$ ) is a triple $(S, R, V$ ), where $S$ is a nonempty set of states, $R: \mathcal{I} \rightarrow \wp(S \times S)$ assigns to every modality $\square_{i}$ a binary relation $R_{i}$ on $S$, and $V$ : PROP $\rightarrow S$ is a valuation which associates with every propositional variable a set of states where it is true.

Definition 2 (satisfaction). For a given formula $\alpha$, the truth of it in, or its satisfaction by, a model $M=(S, R, V)$ with a designated state $s$, denoted $M, s \models \alpha$, is defined inductively as follows.

$$
\begin{array}{rlrl}
M, s & \models p & & \text { iff } s \in V(p) \\
M, s & \models \neg \varphi & & \text { iff } \text { not }(M, s) \models \varphi \\
M, s & \models(\varphi \rightarrow \psi) & \text { iff } M, s \models \varphi \text { implies } M, s \models \psi \\
M, s & \models \square_{i} \varphi & \text { iff for all } t \in S, \text { if }(s, t) \in R_{i} \text { then } M, t \models \varphi \\
M, s & \models \cap_{I} \varphi & \text { iff for all } t \in S, \text { if }(s, t) \in \bigcap_{i \in I} R_{i} \text { then } M, t \models \varphi \\
M, s & \models \uplus_{I} \varphi & \text { iff for all } t \in S, \text { if }(s, t) \in \biguplus_{i \in I} R_{i} \text { then } M, t \models \varphi \\
\text { where } \biguplus_{i \in I} R_{i} \text { is the transitive closure of } \bigcup_{i \in I} R_{i} .
\end{array}
$$

${ }^{4}$ Although the symbol $\biguplus$ is sometimes used for disjoint union, we repurpose it here for transitive closure of union. 
Thus, the intersection modalities are interpreted by taking the intersection, and the union $^{+}$modalities by taking the transitive closure of the union. We use "union ${ }^{+}$modalities" as a short name to avoid the more awkward "transitive closure of union modalities".

Given a formula $\varphi$ and a class $\mathscr{C}$ of models, we say $\varphi$ is valid in $\mathscr{C}$ iff $\varphi$ is valid in all models of $\mathscr{C}$. We usually do not choose a class of models arbitrarily, but are rather interested in those based on a certain set of conditions over the binary relations in a model. Such conditions are often called frame conditions. In this paper we are going to focus on some of the most well known frame conditions (see, e.g., [5]). These conditions are seriality, reflexivity, symmetry, transitivity and Euclidicity. It is well known that these frame conditions are characterized by the formulas D $\left(\square_{i} \varphi \rightarrow \neg \square_{i} \neg \varphi\right)$, T $\left(\square_{i} \varphi \rightarrow \varphi\right)$, B $\left(\neg \varphi \rightarrow \square_{i} \neg \square_{i} \varphi\right)$, 4 $\left(\square_{i} \varphi \rightarrow \square_{i} \square_{i} \varphi\right)$ and $5\left(\neg \square_{i} \varphi \rightarrow \square_{i} \neg \square_{i} \varphi\right)$, respectively. With respect to different combinations of these frame conditions, normal modal logics K, D (a.k.a. KD), T (a.k.a. KT), B (a.k.a. KTB), S4 (a.k.a., KT4) and S5 (a.k.a. KT5) based on the language $\mathcal{L}$ are well studied in the literature. We shall refer an "S5 model" to a Kripke model in which the binary relation is an equivalence relation, and likewise for a D, T, B or S4 model.

In this paper we will focus on the counterpart logics over the languages $\mathcal{L}^{\cap}$ and $\mathcal{L}^{\cap \uplus}$, and they will be named in a comprehensive way as follows:

$$
\begin{aligned}
& \mathrm{K}^{\cap}, \mathrm{D}^{\cap}, \mathrm{T}^{\cap}, \mathrm{B}^{\cap}, \mathrm{S} 4^{\cap}, \mathrm{S} 5^{\cap}, \\
& \mathrm{K}^{\cap \uplus}, \mathrm{D}^{\cap \uplus}, \mathrm{T}^{\cap \uplus}, \mathrm{B}^{\cap \uplus}, \mathrm{S} 4^{\cap \uplus}, \mathrm{S} 5^{\cap \uplus} \text {. }
\end{aligned}
$$

There are well known applications of these logics, for example are $S 5^{\cap}$ and $\mathrm{S} 5^{\cap \uplus}$ (under the restriction that $\mathcal{I}$ is finite) well known as S5D and S5CD respectively in the area of epistemic logic. The logics $\mathrm{K}^{\cap}$ and $\mathrm{S} 4^{\cap}$ are known as $\mathcal{A L C}(\cap)$ (i.e., $\mathcal{A L C}$ with role intersection) and $\mathcal{S}(\cap)$ (where $\mathcal{S}$ is $\mathcal{A L C}$ with role transitiv-

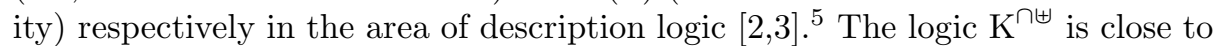
propositional dynamic logic with intersection (IPDL) 9] or the description logic $\mathcal{A L C}(\cap, \cup, *)$, and similarly, $\mathrm{S} 4^{\cap \uplus}$ close to $\mathcal{S}(\cap, \cup, *) 6$

The minimal logic $\mathrm{K}$ can be axiomatized by the system $\mathbf{K}$ composed of the following axiom (schemes) and rules (where $\varphi, \psi \in \mathcal{L}$ and $i \in \mathcal{I}$ ):

(PC) all instances of all propositional tautologies

\footnotetext{
${ }^{5}$ The subscript $i$ of a unary modal operator $\square_{i}$ typically stands for an agent in epistemic logic or a role in description logic. In epistemic logic, a finite number of agents is assumed, and the intersection modality (i.e., a distributed knowledge operator) is an arbitrary intersection over a finite domain. In description logic, the number of roles are typically unbounded, but the intersection is binary, which is in effect equivalent to finite intersection.

${ }^{6}$ There are two major differences however. First, the Kleene star in both logics are the reflexive-transitive closure, and we consider the transitive closure which is denoted by a "+" in the symbol $\uplus$. Second, $\uplus_{I}$ is a compound modality (union and then take the transitive closure), while in those logics the Kleene star is separated from the union, and as a result, the Kleene star applies to the intersection as well, which we do not consider here.
} 
(MP) from $(\varphi \rightarrow \psi)$ and $\varphi$ infer $\psi$

(K) $\square_{i}(\varphi \rightarrow \psi) \rightarrow\left(\square_{i} \varphi \rightarrow \square_{i} \psi\right)$

(N) from $\varphi$ infer $\square_{i} \varphi$

Axiomatizations for D, T, B , S4 and S5, which are named D, T, B, S4 and S5 respectively, can be obtained by adding characterization axioms to $\mathbf{K}$. In more detail, $\mathbf{D}=\mathbf{K} \oplus \mathrm{D}, \mathbf{T}=\mathbf{K} \oplus \mathrm{T}, \mathbf{B}=\mathbf{T} \oplus \mathrm{B}, \mathbf{S 4}=\mathbf{T} \oplus 4$ and $\mathbf{S 5}=\mathbf{T} \oplus 5$, where the symbol $\oplus$ means combining the axioms and rules of the two parts. Details can be found in standard modal logic textbooks (see, e.g., [5]4]).

A logic extended with the intersection modality is typically axiomatized by adding axioms and rules to the corresponding logic without intersection. The axioms and rules to be added are in total called the characterization of intersection, and depends on which logic we are dealing with. Similarly we can define the characterization of the transitive closure of union, which can be made independent to the concrete logic (will be made clear in Section 4).

Characterizations of intersection and transitive closure of union can be found in the literature for some of the logics, including $\mathrm{K}^{\cap}, \mathrm{T}^{\cap}, \mathrm{S} 4^{\cap}, \mathrm{S} 5^{\cap}$ and $\mathrm{S} 5^{\cap \uplus}$ in epistemic logic (see 7111/2]). In particular, for the base logic S5, the characterizations are Int(S5) and $\mathbf{U n}(\mathbf{S} 5)$, respectively:

$\operatorname{Int}(\boldsymbol{S 5})$ characterization of intersection in $\mathbf{S} \mathbf{5}^{\cap}$ and $\mathbf{S} \mathbf{5}^{\cap \uplus}(\mathrm{D} \cap, 4 \cap, \mathrm{B} \cap$ and $\mathrm{N} \cap$ are not necessary in the sense that they are derivable):

$-(\mathrm{K} \cap) \cap_{I}(\varphi \rightarrow \psi) \rightarrow\left(\cap_{I} \varphi \rightarrow \cap_{I} \psi\right)$

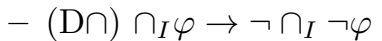

$-(\mathrm{T} \cap) \cap_{I} \varphi \rightarrow \varphi$

$-(4 \cap) \cap_{I} \varphi \rightarrow \cap_{I} \cap_{I} \varphi$

$-(\mathrm{B} \cap) \neg \varphi \rightarrow \cap_{I} \neg \cap_{I} \varphi$

$-(5 \cap) \neg \cap_{I} \varphi \rightarrow \cap_{I} \neg \cap_{I} \varphi$

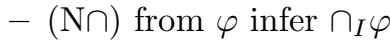

$-(\cap 1) \square_{i} \varphi \leftrightarrow \cap_{\{i\}} \varphi$

$-(\cap 2) \cap_{I} \varphi \rightarrow \cap_{J} \varphi$, if $I \subseteq J$

$\boldsymbol{U n}(\boldsymbol{S} 5)$ characterization of transitive closure of union in $\mathbf{S} \mathbf{5}^{\cap \uplus},(\mathrm{D} \uplus, \mathrm{T} \uplus, 4 \uplus$, $\mathrm{B} \uplus, 5 \uplus$ and $\mathrm{N} \uplus$ are not necessary in the sense that they are derivable):

- $(\mathrm{K} \uplus) \uplus_{I}(\varphi \rightarrow \psi) \rightarrow\left(\uplus_{I} \varphi \rightarrow \uplus_{I} \psi\right)$

$-(\mathrm{D} \uplus) \uplus_{I} \varphi \rightarrow \neg \uplus_{I} \neg \varphi$

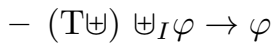

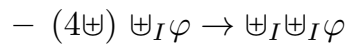

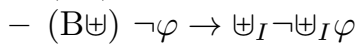

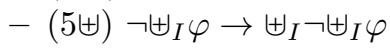

- $(\mathrm{N} \uplus)$ from $\varphi$ infer $\uplus_{I} \varphi$

- $(\uplus 1) \uplus_{I} \varphi \rightarrow \square_{i}\left(\varphi \wedge \uplus_{I} \varphi\right)$, if $i \in I$

- $(\uplus 2)$ from $\varphi \rightarrow \bigwedge_{i \in I} \square_{i}(\varphi \wedge \psi)$ infer $\varphi \rightarrow \uplus_{I} \psi$ 
It is known that the axiomatization $\mathbf{S 5} \cap=\mathbf{S 5} \oplus \mathbf{I n t}(\mathbf{S 5})$ is sound and complete for the logic $\mathbf{S} 5^{\cap}$, and $\mathbf{S} 5^{\cap \uplus}=\mathbf{S} 5^{\cap} \oplus \mathbf{U n}(\mathbf{S 5})$ is sound and complete for the logic $S 5^{\cap \uplus}$ (see, e.g., 7 ), in the case that $\mathcal{I}$ is finite. However, since the intersection and union ${ }^{+}$modalities are interpreted as operations over relations for standard box operators, their properties change in accordance with those for standard boxes. As a result, the characterization axioms and rules vary for weaker logics. We shall look into this in the following sections. First we define some basic terminology that will be useful.

Definition 3 (paths, (proper) initial segments, rest, tail). Given a model $M=(S, R, V)$, a path of $M$ is a finite nonempty sequence $\left\langle s_{0}, I_{0}, \ldots, I_{n-1}, s_{n}\right\rangle$ where: (i) $s_{0}, \ldots, s_{n} \in S$, (ii) $I_{0}, \ldots, I_{n-1}$ are indices, and (iii) for all $x=$ $0, \ldots, n-1,\left(s_{x}, s_{x+1}\right) \in \bigcap_{i \in I} R_{i}$.

Given two paths $s=\left\langle s_{0}, I_{0}, \ldots, I_{m-1}, s_{m}\right\rangle$ and $t=\left\langle t_{0}, J_{0}, \ldots, J_{n-1}, t_{n}\right\rangle$ of a model,

- We say $s$ is an initial segment of $t$, denoted $s \preceq t$, if $m \leq n, s_{x}=t_{x}$ for all $x=0, \ldots, m$, and $I_{y}=J_{y}$ for all $y=0, \ldots, m-1$, and we say that $t$ extends $s$ with $\left\langle J_{m}, t_{m+1}, \ldots, J_{n-1}, t_{n}\right\rangle$;

- We say $s$ is a proper initial segment of $t$, denoted $s \prec t$, if the former is an initial segment of the latter and $m<n$;

- We write tail $(s)$ for $s_{m}$, and similarly tail $(t)$ for $t_{n}$;

- When $s$ is an initial segment of $t$, we write $t \backslash s$ to stand for the path $\left\langle t_{m}, J_{m}, \ldots, J_{n-1}, t_{n}\right\rangle$. Note that tail $(s)$ is kept in $t \backslash s$, and when $s=t$, we have $t \backslash s=\left\langle t_{n}\right\rangle$.

Given a natural number $i$, a path $s=\left\langle s_{0}, I_{0}, \ldots, I_{n-1}, s_{n}\right\rangle$ is called:

- An $i$-path, if $i$ appears in all the indices of the path, i.e., $i \in \bigcap_{x=0}^{n-1} I_{x}$ (note that a path of length 1 , such as $\left\langle s_{0}\right\rangle$, is trivially an $i$-path).

- An I-path, where I is an index, if I is a subset of all the indices of the path, i.e., $I \subseteq \bigcap_{x=0}^{n-1} I_{x}$.

\section{Logics over $\mathcal{L}^{\cap}$}

In this section we study the logics over the language $\mathcal{L}^{\cap}$, namely, $\mathrm{K}^{\cap}, \mathrm{D}^{\cap}, \mathrm{T}^{\cap}$, $\mathrm{B}^{\cap}, \mathrm{S} 4^{\cap}$ and $\mathrm{S} 5^{\cap}$, which means that in this section a "formula" stands for a formula of $\mathcal{L}^{\cap}$, and a "logic" without further explanation refers to one of the six. We shall provide a general method for proving completeness in these logics.

The axiomatization $\mathbf{L}$ we will provide for a logic $\mathbf{L}$ is an extension of the axiomatization for the corresponding logic without intersection, with the characterization of intersection. The characterization of intersection is dependent on the frame conditions. For a given class of models, the characterization of intersection is listed below:

$-\operatorname{Int}(\mathbf{K})=\operatorname{Int}(\mathbf{D})=\{\mathrm{K} \cap, \mathrm{N} \cap, \cap 1, \cap 2\}$

$-\operatorname{Int}(\mathbf{T})=\{\mathrm{K} \cap, \mathrm{T} \cap, \mathrm{N} \cap, \cap 1, \cap 2\}$ 
$-\operatorname{Int}(B)=\{\mathrm{K} \cap, \mathrm{T} \cap, \mathrm{B} \cap, \mathrm{N} \cap, \cap 1, \cap 2\}$
$-\operatorname{Int}(\mathbf{S 4})=\{\mathrm{K} \cap, \mathrm{T} \cap, 4 \cap, \mathrm{N} \cap, \cap 1, \cap 2\}$
$-\operatorname{Int}(\mathbf{S 5})=\{\mathrm{K} \cap, \mathrm{T} \cap, 5 \cap, \mathrm{N} \cap, \cap 1, \cap 2\}$

where $\operatorname{Int}(\mathbf{K})$ is the characterization of intersection for the class of all models, $\operatorname{Int}(\mathbf{D})$ for the class of all D models, $\operatorname{Int}(\mathbf{T})$ for the class of all T models, and so on. We stress that $\mathrm{D} \cap$ is not included in $\operatorname{Int}(\mathbf{D})$ : it is in fact invalid in $\mathrm{D}^{\bigcap}$.

By adding the characterization of intersection to the axiomatization of a logic, we get an axiomatization for the corresponding logic over $\mathcal{L}^{\cap}$. To be precise, we list the axiomatizations as follows:

$$
\begin{aligned}
\mathbf{K}^{\cap} & =\mathbf{K} \oplus \mathbf{I n t}(\mathbf{K}) \\
\mathbf{D}^{\cap} & =\mathbf{D} \oplus \mathbf{I n t}(\mathbf{D}) \\
\mathbf{T}^{\cap} & =\mathbf{T} \oplus \mathbf{I n t}(\mathbf{T}) \\
\mathbf{B}^{\cap} & =\mathbf{B} \oplus \mathbf{I n t}(\mathbf{B}) \\
\mathbf{S}^{\cap} & =\mathbf{S} \mathbf{I} \oplus \mathbf{I n t}(\mathbf{S} 4) \\
\mathbf{S} 5^{\cap} & =\mathbf{S} 5 \oplus \mathbf{I n t}(\mathbf{S} 5)
\end{aligned}
$$

It is not hard to verify that all the above axiomatizations are sound in their corresponding logic, respectively.

Some of the above axiomatizations, in particular, $\mathbf{K}^{\cap}, \mathbf{T}^{\cap}, \mathbf{S} \mathbf{4}^{\cap}$ and $\mathbf{S} 5^{\cap}$, are given in [7. An outline of proof of completeness is also found there, without details. Detailed proofs can be found for certain special cases, such as the $\mathbf{S 5}^{\cap}$ with only a single intersection modality for the set of all agents (which is assumed to be finite) [6]. A general and detailed proof based on this technique can be found in 13. The proof goes through an unraveling-folding procedure, mentioned already in the introduction. Due to the subtleties in the unraveling and folding processes, it is diffucult to apply this technique directly to new logics, as definitions may differ already from the beginning (for example, the definition of a path is already different) all the way to the very end of the procedure. This in general becomes an obstacle for developers of new logics with the intersection modality.

We introduce a simpler method for proving completeness, that can easily be adapted to a range of different logics. This is a relatively simple variant of the standard canonical model method. For each of the logics L mentioned above, with corresponding axiomatization $\mathbf{L}$, we shall show that $\mathbf{L}$ is a complete axiomatization of $\mathrm{L}$, which is equivalent to finding an $\mathrm{L}$ model for every $\mathbf{L}$ consistent set of formulas. The model we are going to build is called a standard model.

Let $\mathrm{MCS}^{\mathbf{L}}$ be the set of all maximal $\mathbf{L}$-consistent sets of $\mathcal{L}^{\cap}$-formulas.7 Given $\mathbf{L}$, given an index $I$, we shall write $\triangleright_{I}$ to stand for a binary relation on $\operatorname{MCS}^{\mathbf{L}}$, such that $\Phi \triangleright_{I} \Psi$ iff for all $\varphi, \cap_{I} \varphi \in \Phi$ implies $\varphi \in \Psi$. This type of relations is typically used in the definition of a canonical model (as found in modal textbooks), and are sometimes called canonical relations. We easily get the following proposition.

\footnotetext{
${ }^{7}$ We refer to a modal logic textbook, say 4, for a definition of a (maximal) consistent set of formulas.
} 
Proposition 4 (canonicity). For any index I, the canonical relation $\triangleright_{I}$ on $M C S^{L}$ is:

1. Serial, if $I$ is singleton and $\boldsymbol{L}$ is $\boldsymbol{D}^{\cap}$;

2. Reflexive, if $\boldsymbol{L}$ is $\boldsymbol{T}^{n}$;

3. Reflexive and symmetric, if $\boldsymbol{L}$ is $\boldsymbol{B}^{\cap}$;

4. Reflexive and transitive, if $\boldsymbol{L}$ is $\boldsymbol{S}_{4}$;

5. An equivalence relation, if $\boldsymbol{L}$ is $\boldsymbol{S} \boldsymbol{5}^{n}$.

We note that if $\boldsymbol{L}$ is $\boldsymbol{D}^{\bigcap}, \triangleright_{I}$ is not necessarily serial when $I$ is not a singleton. Moreover,

6. $\triangleright_{J} \subseteq \triangleright_{I}$, for any index $J \supseteq I$.

Definition 5 (canonical paths). Given an axiomatization $\boldsymbol{L}$, a canonical path for $\boldsymbol{L}$ is a sequence $\left\langle\Phi_{0}, I_{0}, \ldots, I_{n-1}, \Phi_{n}\right\rangle$ such that:

(i) $\Phi_{0}, \ldots, \Phi_{n} \in M C S^{L}$,

(ii) $I_{0}, \ldots, I_{n-1}$ are indices, and

(iii) for all $x=0, \ldots, n-1,\left(s_{x}, s_{x+1}\right) \in \triangleright_{I_{x}}$.

Initial segments, tail(s), $i$-paths, I-paths, and so on, are defined exactly like for paths in a model (Def. [3).

The standard models we will define for these logics are a bit different from the canonical model for a standard modal logic. As mentioned existing proofs are based on transforming the canonical model to a treelike model. We will construct a treelike model directly: in the standard model for a logic L, a state will be a canonical path for $\mathbf{L}$. However, the binary relations in a standard model is dependent on the concrete logic we focus on. We now first define these binary relations and then introduce the definition of a standard model.

Definition 6 (standard relations). Given a logic $L$ with its axiomatization $\boldsymbol{L}$, we define $\mathrm{R}^{L}$ as follows. For any $i \in \mathcal{I}, \mathrm{R}_{i}^{L}$ is the binary relation on the set of canonical paths for $\boldsymbol{L}$, called the standard relation for $i$, such that:

- When $L$ is $K^{\cap}$ or $D^{\cap}$ : for all canonical paths $s$ and $t$ for $\boldsymbol{L},(s, t) \in \mathrm{R}_{i}^{L}$ iff $t$ extends $s$ with $\langle I, \Phi\rangle$ for some $I \ni i$ and $\Phi \in M C S^{L}$;

- When $L$ is $T^{\cap}$ : for all canonical paths $s$ and $t$ for $\boldsymbol{T}^{\bigcap},(s, t) \in \mathrm{R}_{i}^{T^{\cap}}$ iff $t=s$ or $t$ extends $s$ with $\langle I, \Phi\rangle$ for some $I \ni i$ and $\Phi \in M C S^{T^{n}}$;

- When $L$ is $B^{\cap}$ : for all canonical paths $s$ and $t$ for $\boldsymbol{B}^{\bigcap},(s, t) \in \mathrm{R}_{i}^{B^{\bigcap}}$ iff (i) $t=s$ or (ii) $s$ extends $t$ with $\langle I, \Phi\rangle$ or (iii) $t$ extends $s$ with $\langle I, \Phi\rangle$ for some $i \in I$ and $\Phi \in M C S^{B^{\bigcap}}$;

- When $L$ is $S_{4}^{\cap}$ : for all canonical paths $s$ and $t$ for $\boldsymbol{S 4}_{4}^{\cap},(s, t) \in \mathrm{R}_{i}^{S 4^{\cap}}$ iff $s$ is an initial segment of $t$ and $t \backslash s$ is a canonical $i$-path;

- When $L$ is $S 5^{\curvearrowleft}$ : for all canonical paths $s$ and $t$ for $\boldsymbol{S} \boldsymbol{5}^{\cap},(s, t) \in \mathrm{R}_{i}^{S 5^{\curvearrowleft}}$ iff (i) $s$ and $t$ have a common initial segment $u$, and (ii) both $s \backslash u$ and $t \backslash u$ are canonical $i$-paths.

Definition 7 (standard models). Given a logic $L$, the standard model for $L$ is a tuple $\mathrm{M}^{L}=(\mathrm{S}, \mathrm{R}, \mathrm{V})$ such that: 
- $\mathrm{S}$ is the set of all canonical paths for $\boldsymbol{L}$; its elements are called states of $\mathrm{M}^{L}$.

$-\mathrm{R}=\mathrm{R}^{L}$.

- For any propositional variable $p, \mathrm{~V}(p)=\{s \in \mathrm{S} \mid p \in \operatorname{tail}(s)\}$.

Lemma 8 (standardness). The following hold:

1. $\mathrm{M}^{K^{\bigcap}}$ is a Kripke model;

2. $\mathrm{M}^{D^{n}}$ is a D model;

3. $\mathrm{M}^{T^{\cap}}$ is a T model;

4. $\mathrm{M}^{B^{\cap}}$ is a $B$ model;

5. $\mathrm{M}^{S 4^{n}}$ is an $S_{4}$ model;

6. $\mathrm{M}^{S 5^{n}}$ is an $S 5$ model.

Lemma 9 (existence). For any logic $L$, any state $s$ of $\mathrm{M}^{L}$, and any index $I$, if $\cap_{I} \varphi \notin$ tail $(s)$ then there is a state $t$ of $\mathrm{M}^{L}$ such that $(s, t) \in \bigcap_{i \in I} \mathrm{R}_{i}^{L}$ and $\varphi \notin \operatorname{tail}(t)$.

Proof. Let $s$ be a state of $\mathrm{M}^{\mathrm{L}}$ and $\cap_{I} \varphi \notin$ tail $(s)$. So $\neg \cap_{I} \varphi \in$ tail $(s)$. Consider the set $\Phi^{-}=\{\neg \varphi\} \cup\left\{\psi \mid \cap_{I} \psi \in \operatorname{tail}(s)\right\}$. We can show $\Phi^{-}$is $\mathbf{L}$ consistent just as in a classical proof of the existence lemma (see, e.g., [4). We can then extend it into a maximal consistent set $\Phi$ of formulas using the Lindenbaum construction. Since $\neg \varphi \in \Phi, \varphi \notin \Phi$. Let $t$ be $s$ extended with $\langle I, \Phi\rangle$. By definition it is clear that $\varphi \notin \operatorname{tail}(t)$ and for all $\mathrm{L},(s, t) \in \bigcap_{i \in I} \mathrm{R}_{i}^{\mathrm{L}}$ (since $s \mathrm{R}_{i}^{\mathrm{L}} t$ for all $i \in I$ ).

Lemma 10 (truth). Given a logic L, a formula $\varphi$, and a state $s$ of $\mathrm{M}^{L}$,

$$
\mathrm{M}^{L}, s \models \varphi \quad \text { iff } \quad \varphi \in \operatorname{tail}(s) .
$$

Proof. The proof is by induction on $\varphi$. The atomic case is by definition. Boolean cases are easy to show. Interesting cases are for the modalities $\square_{i}(i \in \mathcal{I})$ and $\cap_{I}(I$ is an index $)$. We start with the case for $\cap_{I} \psi$.

$$
\begin{aligned}
& \mathrm{M}^{\mathrm{L}}, s \models \cap_{I} \psi \\
\Leftrightarrow & \text { for all } t, \text { if }(s, t) \in \bigcap_{i \in I} \mathrm{R}_{i}^{\mathrm{L}} \text { then } \mathrm{M}^{\mathrm{L}}, t \models \psi \\
\Leftrightarrow & \text { for all } t, \text { if }(s, t) \in \bigcap_{i \in I} \mathrm{R}_{i}^{\mathrm{L}} \text { then } \psi \in \operatorname{tail}(t) \\
\Rightarrow & \cap_{I} \psi \in \text { tail }(s)
\end{aligned}
$$

For the converse direction of the last step, suppose $\cap_{I} \psi \in \operatorname{tail}(s)$ and assume towards a contradiction that there is a state $t$ such that $(s, t) \in \bigcap_{i \in I} \mathrm{R}_{i}^{\mathrm{L}}$ and $\psi \notin \operatorname{tail}(t)$.

- If $\mathrm{L}$ is $\mathrm{K}^{\cap}$ or $\mathrm{D}^{\cap}$, it must be that $t$ extends $s$ with $\langle J, \Phi\rangle$ for $J \supseteq I$ and $\Phi \in \mathrm{MCS}^{\mathbf{L}}$. By definition tail $(s) \triangleright_{J}$ tail $(t)$, and by Proposition 416, we have tail $(s) \triangleright_{I}$ tail $(t)$. Therefore $\psi \in$ tail $(t)$, which leads to a contradiction.

- If $\mathrm{L}$ is $\mathrm{T}^{\cap}$, we face an extra case compared with the above, namely $s=t$. A contradiction can be reached by applying the axiom $\mathrm{T} \cap$.

- If $\mathrm{L}$ is $\mathrm{B}^{\cap}$, there are three cases: (i) $t=s$ or (ii) $s=\langle t, J, \Phi\rangle$ or (iii) $t=$ $\langle s, J, \Phi\rangle$ where $J \supseteq I$ and $\Phi \in \mathrm{MCS}^{\mathrm{B}}$. Case (i) can be shown similarly to the case when $L$ is $T^{\bigcap}$, and case (iii) to the case when $L$ is $K^{\cap}$ or $D^{\cap}$. For case (ii), it is important to observe that $\triangleright_{I}$ is symmetric (Proposition 413) and $\triangleright_{J} \subseteq \triangleright_{I}$ (Proposition 466). 
- If L is $\mathrm{S} 4^{\cap}, s$ must be an initial segment of $t$ and $t \backslash s$ is an $I$-path. We get $\operatorname{tail}(s) \triangleright_{I}$ tail $(t)$ by Proposition 46 and the reflexivity and transitivity of $\triangleright_{I}$ (Proposition 44). Therefore $\psi \in$ tail $(t)$ which leads to a contradiction.

- If L is $\mathrm{S} 5 \cap$, then $s$ and $t$ have a common initial segment $u$, and $s \backslash u$ and $t \backslash u$ are both $I$-paths. For special cases when one of $s$ and $t$ is an initial segment of the other, it can be shown like in the case when $\mathrm{L}$ is $\mathrm{S} 4^{\cap}$. The interesting case is when $s$ and $t$ really fork, in this case we can show both tail $(s) \triangleright_{I} \operatorname{tail}(u)$ and tail $(u) \triangleright_{I}$ tail $(t)$ by transitivity and symmetry of $\triangleright_{I}$ (Proposition 45) and Proposition 46, so that tail $(s) \triangleright_{I} \operatorname{tail}(t)$. Then $\psi \in \operatorname{tail}(t)$, which leads to a contradiction.

Finally, the case for $\square_{i} \psi$ :

$$
\begin{aligned}
& \mathrm{M}^{\mathrm{L}}, s \models \square_{i} \psi \\
\Leftrightarrow & \mathrm{M}^{\mathrm{L}}, s \models \cap_{\{i\}} \psi(\text { validity of } \cap 1) \\
\Leftrightarrow & \left.\cap_{\{i\}} \psi \in \operatorname{tail}(s) \quad \text { (special case of } \cap_{I} \psi\right) \\
\Leftrightarrow & \square_{i} \psi \in \operatorname{tail}(s) \quad(\text { axiom } \cap 1)
\end{aligned}
$$

Theorem 11 (strong completeness). Given $L \in\left\{K^{\cap}, D^{\cap}, T^{\cap}, B^{\cap}, S 4^{\cap}, S 5^{\cap}\right\}$ and its axiomatization $\boldsymbol{L}$, for any $\Phi \subseteq \mathcal{L}^{\cap}$ and $\varphi \in \mathcal{L}^{\cap}$, if $\Phi \models \varphi$, then $\Phi \vdash_{\boldsymbol{L}} \varphi$.

Proof. Suppose $\Phi \nvdash_{\mathbf{L}} \varphi$. It follows that $\Phi \cup\{\neg \varphi\}$ is $\mathbf{L}$ consistent. Extend it to be a maximal set $\Psi$, then $\langle\Psi\rangle$ is a canonical path. By the truth lemma, for any formula $\psi$, we have $\mathrm{M},\langle\Psi\rangle \models \psi$ iff $\psi \in \Psi$. It follows that $\Psi$ is satisfiable, which leads to $\Phi \not \forall \varphi$.

\section{Logics over $\mathcal{L}^{\cap \uplus}$}

In this section we study the logics with both the intersection and union ${ }^{+}$modalities. The language is set to be $\mathcal{L}^{\cap \uplus}$ in this section, and by a "logic" without further explanation we mean one of $\mathrm{K}^{\cap \uplus}, \mathrm{D}^{\cap \uplus}, \mathrm{T}^{\cap \uplus}, \mathrm{B}^{\cap \uplus}, \mathrm{S} 4^{\cap \uplus}$ or $\mathrm{S} 5^{\cap \uplus}$.

Compared with the characterization of intersection, that of transitive closure of union is better behaved:

$$
\mathbf{U n}(\mathbf{K})=\mathbf{U n}(\mathbf{D})=\mathbf{U n}(\mathbf{T})=\mathbf{U n}(\mathbf{B})=\mathbf{U n}(\mathbf{S} 4)=\mathbf{U n}(\mathbf{S} 5)=\{K \uplus, \uplus 1, \uplus 2\}
$$

These axioms are not new, as e.g., is found in [7, although as far as we know they have not been studied as additions to D and B in the literature. For simplicity we write Un this set of axioms. Additional axioms for union ${ }^{+}$corresponding to specific frame conditions can be derived in specific logic systems. For instance, $\mathrm{D} \uplus$ is a theorem of $\mathbf{D} \oplus \mathbf{U n}$.

By adding to the axiomatization of a logic over $\mathcal{L}^{\cap}$ the characterization of union $^{+}$, we get a sound axiomatization for the corresponding logic over $\mathcal{L}^{\cap \uplus}$. To 
make it precise, we list the axiomatizations as follows:

$$
\begin{aligned}
& \mathbf{K}^{\cap \uplus}=\mathbf{K}^{\cap} \oplus \mathbf{U n} \\
& \mathbf{D}^{\cap \uplus}=\mathbf{D}^{\cap} \oplus \mathbf{U n} \\
& \mathbf{T}^{\cap \uplus}=\mathbf{T}^{\cap} \oplus \mathbf{U n} \\
& \mathbf{B}^{\cap \uplus}=\mathbf{B}^{\cap} \oplus \mathbf{U n} \\
& \mathbf{S} 4^{\cap \uplus}=\mathbf{S} 4^{\cap} \oplus \mathbf{U n} \\
& \mathbf{S} 5^{\cap \uplus}=\mathbf{S} 5^{\cap} \oplus \mathbf{U n}
\end{aligned}
$$

In this section we will make extensive references to the names of logics and axiomatizations, and for simplicity we shall call a tuple $\sigma=(\mathrm{L}, \mathbf{L}, \alpha, \iota)$ a signature, when $\mathrm{L}$ is one of the logics $\mathrm{K}^{\cap \uplus}, \mathrm{D}^{\cap \uplus}, \mathrm{T}^{\cap \uplus}, \mathrm{B}^{\cap \uplus}, \mathrm{S} 4^{\cap \uplus}$ and $\mathrm{S} 5^{\cap \uplus}, \mathbf{L}$ is the corresponding axiomatization for $\mathrm{L}, \alpha$ is a formula of $\mathcal{L}^{\cap \uplus}$, and $\iota$ is an index such that every index occurring in $\alpha$ is a subset of $\iota$. Moreover, we write $\sigma(\mathrm{K})$ when the $\mathrm{L}$ in $\sigma$ is $\mathrm{K}$, and similarly for $\sigma(\mathrm{D}), \sigma(\mathrm{T}), \sigma(\mathrm{B}), \sigma(\mathrm{S} 4)$ and $\sigma(\mathrm{S} 5)$.

Definition 12 (closure). Given a signature $\sigma=(L, \boldsymbol{L}, \alpha, \iota)$, the $\sigma$-closure, denoted $\mathrm{cl}(\sigma)$, is the minimal set of formulas satisfying the following conditions:

1. $\alpha \in \operatorname{cl}(\sigma)$;

2. If $\varphi \in \operatorname{cl}(\sigma)$, then all the subformulas of $\varphi$ are also in $\operatorname{cl}(\sigma)$;

3. If $\varphi$ does not start with a negation symbol and $\varphi \in \operatorname{cl}(\sigma)$, then $\neg \varphi \in \operatorname{cl}(\sigma)$;

4. For any $i \in \iota, \cap_{\{i\}} \varphi \in \operatorname{cl}(\sigma)$ if and only if $\square_{i} \varphi \in \operatorname{cl}(\sigma)$;

5. For any $I$ and $J$ such that $I \subset J \subseteq \iota$, if $\cap_{I} \varphi \in \operatorname{cl}(\sigma)$ then $\cap_{J} \varphi \in \operatorname{cl}(\sigma)$;

6. For any $I$ and $J$ such that $I \subset J \subseteq \iota$, if $\uplus_{I} \varphi \in \operatorname{cl}(\sigma)$ then $\left\{\cap_{J} \uplus_{I} \varphi \mid I \cap J \neq\right.$ $\emptyset\} \subseteq \operatorname{cl}(\sigma)$.

It is not hard to verify that $\operatorname{cl}(\sigma)$ is finite and nonempty for any signature $\sigma$. Given $\sigma=(\mathrm{L}, \mathbf{L}, \alpha, \iota)$, a set of formulas is said to be maximal $\boldsymbol{L}$-consistent in $\operatorname{cl}(\sigma)$, if it is (i) a subset of $\operatorname{cl}(\sigma)$, (ii) $\mathbf{L}$-consistent and (iii) maximal in $\operatorname{cl}(\sigma)$ (i.e., any proper superset which is a subset of $\operatorname{cl}(\sigma)$ is inconsistent). We write $\mathrm{MCS}^{\sigma}$ for the set of all maximal L-consistent sets of formulas in $\operatorname{cl}(\sigma)$.

Now we extend the canonical relations to the finitary case. Given a signature $\sigma$ and an index $I$, we may try to define a canonical relation $\triangleright_{I}$ to be a binary relation on $\operatorname{MCS}^{\sigma}$, such that $\Phi \triangleright_{I} \Psi$ iff for all $\varphi, \cap_{I} \varphi \in \Phi$ implies $\varphi \in \Psi$, like we did for the logics over $\mathcal{L}^{\cap}$. But there are subtleties regarding the closure. For example, transitivity may be lost in $\mathrm{S} 4^{\cap \uplus}$, if $\cap_{I} \varphi \in \Phi$ but $\cap_{I} \cap_{I} \varphi \notin \Phi$ in case the latter is not included in the closure. We introduce the formal definition below.

Definition 13 (finitary canonical relation). For a signature $\sigma=(L, L, \alpha, \iota)$ and an index $I \subseteq \iota$, the canonical relation $\triangleright_{I}$ for $\mathrm{L}$ is a binary relation on $M C S^{\sigma}$, such that the following hold for all $\Phi, \Psi \in M C S^{\sigma}$ :

- If $L$ is $K^{\cap \uplus}, D^{\cap \uplus}$ or $T^{\natural \uplus}: \Phi \triangleright_{I} \Psi$ iff $\left\{\varphi \mid \cap_{I} \varphi \in \Phi\right\} \subseteq \Psi$;

- If $L$ is $B^{\cap \uplus}: \Phi \triangleright_{I} \Psi$ iff $\left\{\varphi \mid \cap_{I} \varphi \in \Phi\right\} \subseteq \Psi$ and $\left\{\varphi \mid \cap_{I} \varphi \in \Psi\right\} \subseteq \Phi$;

- If $L$ is $S 4^{\cap \uplus}: \Phi \triangleright_{I} \Psi$ iff $\left\{\cap_{I} \varphi \mid \cap_{I} \varphi \in \Phi\right\} \subseteq\left\{\cap_{I} \varphi \mid \cap_{I} \varphi \in \Psi\right\}$;

- If $L$ is $S 5^{\cap \uplus}: \Phi \triangleright_{I} \Psi$ iff $\left\{\cap_{I} \varphi \mid \cap_{I} \varphi \in \Psi\right\}=\left\{\cap_{I} \varphi \mid \cap_{I} \varphi \in \Psi\right\}$. 
Note that for all the logics, from $\Phi \triangleright_{I} \Psi$ we still get that $\cap_{I} \varphi \in \Phi$ implies $\varphi \in \Psi$, as the criteria above are at least not weaker. We can get the following proposition that is similar to Proposition 4 .

Proposition 14. For any signature $\sigma=(L, \boldsymbol{L}, \alpha, \iota)$ and any index $I \subseteq \iota$, the canonical relation $\triangleright_{I}$ on $M C S^{\sigma}$ is:

1. Serial, if $I$ is singleton and $\boldsymbol{L}$ is $\boldsymbol{D}^{\bigcap \uplus}$;

2. Reflexive, if $\boldsymbol{L}$ is $\boldsymbol{T}^{\mathrm{\uplus}}$;

3. Reflexive and symmetric, if $\boldsymbol{L}$ is $\boldsymbol{B}^{\bigcap \uplus}$;

4. Reflexive and transitive, if $\boldsymbol{L}$ is $\boldsymbol{S}_{4}{ }^{\natural *}$;

5. An equivalence relation, if $\boldsymbol{L}$ is $\boldsymbol{S} 5^{\bigcap \uplus}$.

Moreover,

6. $\triangleright_{J} \subseteq \triangleright_{I}$, for any index $J$ such that $I \subseteq J \subseteq \iota$.

Proof. For the seriality when $I=\{i\}$ : given $\Phi \in \operatorname{MCS}^{\sigma}$ and a formula $\varphi$ such that $\cap_{\{i\}} \varphi \in \Phi$, it suffices to show the existence of a $\Psi \in \operatorname{MCS}^{\sigma}$ such that $\varphi \in \Psi$. This is easy, take $\varphi$ and extend it to be $\mathbf{L}$-maximal in $\operatorname{cl}(\sigma)$, by observing that $\varphi \in \operatorname{cl}(\sigma)$.

For reflexivity, we make use of the axiom $\mathrm{T} \cap$ and the fact that $\operatorname{cl}(\sigma)$ is closed under subformulas.

For the combinations of frame conditions for the axiomatizations $\mathbf{B}^{\bigcap \uplus}, \mathbf{S} \mathbf{4}^{\cap \uplus}$ and $\mathbf{S} 5^{\cap \uplus}$, we can see that they are enforced by the definition of the canonical relation.

Definition 15 (finitary canonical paths). Given a signature $\sigma=(L, \boldsymbol{L}, \alpha, \iota)$, a canonical path for $\boldsymbol{L}$ in $\operatorname{cl}(\sigma)$ is a sequence $\left\langle\Phi_{0}, I_{0}, \ldots, I_{n-1}, \Phi_{n}\right\rangle$ such that:

(i) $\Phi_{0}, \ldots, \Phi_{n} \in M C S^{\sigma}$,

(ii) $I_{0}, \ldots, I_{n-1} \subseteq \iota$, and

(iii) for all $x=0, \ldots, n-1,\left(s_{x}, s_{x+1}\right) \in \triangleright_{I_{x}}$.

Initial segments, tail(s), $i$-paths, I-paths, and so on, are defined exactly like for paths in a model (Def. [3).

Definition 16 (standard relation). Given a signature $\sigma=(L, L, \alpha, \iota)$, for any $i \in \iota$, the standard relation $\mathrm{R}_{i}^{\sigma}$ is a binary relation on the canonical paths for $\boldsymbol{L}$ in $\operatorname{cl}(\sigma)$, such that:

- When $L$ is $K^{\bigcap \uplus}$ or $D^{\bigcap \uplus}$ : for all canonical paths $s$ and $t$ for $\boldsymbol{L}$ in $\operatorname{cl}(\sigma)$, $(s, t) \in \mathrm{R}_{i}^{\sigma}$ iff $t$ extends $s$ with $\langle I, \Phi\rangle$ for $\Phi \in M C S^{\sigma}$ and some index I such that $i \in I \subseteq \iota$;

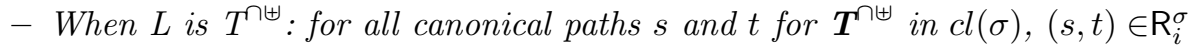
iff $t=s$ or $t$ extends $s$ with $\langle I, \Phi\rangle$ for $\Phi \in M C S^{\sigma}$ and some index I such that $i \in I \subseteq \iota$;

- When $L$ is $B^{\cap \uplus}$ : for all canonical paths $s$ and $t$ for $\boldsymbol{B}^{\cap \uplus}$ in $\operatorname{cl}(\sigma),(s, t) \in \mathrm{R}_{i}^{\sigma}$ iff (i) $t=s$ or (ii) $s=\langle t, I, \Phi\rangle$ or (iii) $t=\langle s, I, \Phi\rangle$ for $\Phi \in M C S^{\sigma}$ and some index $I$ such that $i \in I \subseteq \iota$; 
- When $L$ is $S_{4}^{\cap \uplus}$ : for all canonical paths $s$ and $t$ for $\boldsymbol{S}_{4}^{\cap \uplus}$ in $\operatorname{cl}(\sigma),(s, t) \in \mathrm{R}_{i}^{\sigma}$ iff $s$ is an initial segment of $t$ and $t \backslash s$ is a canonical $i$-path;

- When $L$ is $S 5^{{ }^{\uplus}}$ : for all canonical paths $s$ and $t$ for $\boldsymbol{S} 5^{\natural \uplus}$ in $c l(\sigma),(s, t) \in \mathrm{R}_{i}^{\sigma}$ iff (i) $s$ and thave a common initial segment $u$, and (ii) both $s \backslash u$ and $t \backslash u$ are canonical $i$-paths.

Definition 17 (finitary standard models). Given a signature $\sigma=(L, L, \alpha, \iota)$, the standard model for $\sigma$ is a tuple $\mathrm{M}^{\sigma}=(\mathrm{S}, \mathrm{R}, \mathrm{V})$ such that:

- $\mathbf{S}$ is the set of all canonical paths for $\boldsymbol{L}$ in $\mathrm{cl}(\sigma)$. The elements of $\mathbf{S}$ are called states of $\mathrm{M}^{\sigma}$.

$-\mathrm{R}=\mathrm{R}^{\sigma}$.

- For any propositional variable $p, \mathrm{~V}(p)=\{s \in \mathrm{S} \mid p \in \operatorname{tail}(s)\}$.

Lemma 18 (standardness). Given a signature $\sigma=(L, L, \alpha, \iota)$, the following hold:

1. $\mathrm{M}^{\sigma}$ is a Kripke model;

2. $\mathrm{M}^{\sigma}$ is a $D$ model when $L=D^{\cap \uplus}$ and $\boldsymbol{L}=\boldsymbol{D}^{\bigcap \uplus}$;

3. $\mathrm{M}^{\sigma}$ is a T model when $L=T^{\mathrm{\uplus}}$ and $\boldsymbol{L}=\boldsymbol{T}^{\mathrm{\uplus}}$;

4. $\mathrm{M}^{\sigma}$ is a $B$ model when $L=B^{\cap \uplus}$ and $\boldsymbol{L}=\boldsymbol{B}^{\cap \uplus}$;

5. $\mathrm{M}^{\sigma}$ is an $S_{4}$ model when $L=S_{4}^{\cap \uplus}$ and $\boldsymbol{L}=\boldsymbol{S}_{4}^{\cap \uplus}$;

6. $\mathrm{M}^{\sigma}$ is an $S 5$ model when $L=S 5^{\cap \uplus}$ and $\boldsymbol{L}=\boldsymbol{S} 5^{\bigcap \uplus}$.

Lemma 19 (existence). For any signature $\sigma$, any state $s$ of $\mathrm{M}^{\sigma}$, and any index $I \subseteq \iota$, suppose $\cap_{I} \varphi, \uplus_{I} \varphi \in \operatorname{cl}(\sigma)$. Then,

1. If $\cap_{I} \varphi \notin$ tail(s) then there is a state $t$ of $\mathrm{M}^{\sigma}$ such that $(s, t) \in \bigcap_{i \in I} \mathrm{R}_{i}^{\sigma}$ and $\varphi \notin \operatorname{tail}(t)$.

2. If $\uplus_{I} \varphi \notin$ tail $(s)$ then there is a state $t$ of $\mathrm{M}^{\sigma}$ such that $(s, t) \in \biguplus_{i \in I} \mathrm{R}_{i}^{\sigma}$ and $\varphi \notin \operatorname{tail}(t)$.

Proof. Let $\sigma=(\mathrm{L}, \mathbf{L}, \alpha, \iota)$ and $s$ be a state of $\mathrm{M}^{\sigma}$.

(11) Let $\cap_{I} \varphi \notin$ tail $(s)$. So $\neg \cap_{I} \varphi \in \operatorname{tail}(s)$. Consider the set $\Phi^{-}=\{-\varphi\} \cup\{\psi \mid$ $\left.\cap_{I} \psi \in \operatorname{tail}(s)\right\}$ (where $-\varphi$ is $\psi$ if $\varphi=\neg \psi$, and is $\neg \varphi$ if $\varphi$ is positive). Clearly $\Phi^{-} \subseteq \operatorname{cl}(\sigma)$ and it is not hard to show that it is $\mathbf{L}$ consistent. We can then extend it into a maximal consistent set $\Phi$ of formulas in $\operatorname{cl}(\sigma)$. Since $-\varphi \in \Phi, \varphi \notin \Phi$. Let $t$ be $s$ extended with $\langle I, \Phi\rangle$. By definition it is clear that $\varphi \notin$ tail $(t)$ and $(s, t) \in \bigcap_{i \in I} \mathrm{R}_{i}^{\sigma}$ (since $s \mathrm{R}_{i}^{\sigma} t$ for all $i \in I$ ).

(2) Let $\mathcal{P}$ be the property on the states of $\mathrm{M}^{\sigma}$ such that for any $s, s \in \mathcal{P}$ iff for any $t$, if $(s, t) \in \biguplus_{i \in I} \mathrm{R}_{i}^{\sigma}$ then $\varphi \in \operatorname{tail}(t)$. The equivalent condition is that for any state $s_{0}$ of $\mathrm{M}^{\sigma}, s_{0} \in \mathcal{P}$ iff $\varphi \in \operatorname{tail}\left(s_{n}\right)$ holds for any path $\left\langle s_{0},\left\{i_{0}\right\}, \ldots,\left\{i_{n-1}\right\}, s_{n}\right\rangle$ of $\mathrm{M}^{\sigma}$ with $\left\{i_{0}, \ldots, i_{n-1}\right\} \subseteq I$. Let $\psi=\bigvee_{s \in \mathcal{P}} \widehat{\operatorname{tail}(s)}$ (where $\widehat{\operatorname{tail}(s)}$ is the conjunction of all formulas in tail $(s)$ ). We get the following:

(a) For any $i \in I, \vdash_{\mathbf{L}} \psi \rightarrow \square_{i} \varphi$. First observe that for every $s_{0} \in \mathcal{P}$, any path $\left\langle s_{0},\left\{i_{0}\right\}, \ldots,\left\{i_{n-1}\right\}, s_{n}\right\rangle$ as described above is such that $\varphi \in \operatorname{tail}\left(s_{n}\right)$. As a special case, for any state $s_{1}$, if $\left\langle s_{0},\{i\}, s_{1}\right\rangle$ is a path, namely tail $\left(s_{0}\right) \triangleright_{\{i\}}$ tail $\left(s_{1}\right)$, then $\varphi \in \operatorname{tail}\left(s_{1}\right)$. It follows that $\square_{i} \varphi \in \operatorname{tail}\left(s_{0}\right)$ (for otherwise it violates the first 
clause; just treat $\square_{i}$ to be $\left.\cap_{\{i\}}\right)$. This means that $\square_{i} \varphi$ is a conjunct of every disjunct of $\psi$, and so $\vdash_{\mathbf{L}} \psi \rightarrow \square_{i} \varphi$.

(b) For any $i \in I, \vdash_{\mathbf{L}} \psi \rightarrow \square_{i} \psi$. Suppose towards a contradiction that $\psi \wedge \neg \square_{i} \psi$ is consistent. There must be a disjunct of $\psi$, say $\widehat{\operatorname{tail}(t)}$ (with $t \in \mathcal{P}$ ), such that $\widehat{\operatorname{tail}(t)} \wedge \neg \square_{i} \psi$ is consistent. By properties of $\operatorname{MCS}^{\sigma}$ we have $\vdash_{\mathbf{L}} \bigvee\{\widehat{\Phi} \mid$ $\left.\Phi \in \mathrm{MCS}^{\sigma}\right\}$ (similarly $\widehat{\Phi}$ is the conjunction of formulas in $\Phi$ ). So there must be $\Phi \in \operatorname{MCS}^{\sigma} \backslash\{\operatorname{tail}(s) \mid s \in \mathcal{P}\}$ such that $\widehat{\operatorname{tail}(t)} \wedge \neg \square_{i} \neg \widehat{\Phi}$ is consistent. It follows that tail $(t) \triangleright_{\{i\}} \Phi$. The path $u$ which extends $t$ with $\langle\{i\}, \Phi\rangle$ is such that $(t, u) \in \mathrm{R}_{\{i\}}^{\sigma}$. Since $t \in \mathcal{P}$, we have $u \in \mathcal{P}$ as well. However, this conflicts with the fact that $\Phi \notin\{\operatorname{tail}(s) \mid s \in \mathcal{P}\}$.

Now we show the contraposition of the clause. Suppose $s \in \mathcal{P}$, and we must show $\uplus_{I} \varphi \in \operatorname{tail}(s)$. By (a) and (b) we have $\vdash_{\mathbf{L}} \psi \rightarrow \bigwedge_{i \in I} \square_{i}(\psi \wedge \varphi)$, and then by $\uplus 2$ we have $\vdash_{\mathbf{L}} \psi \rightarrow \uplus_{I} \varphi$. Let $\xi=\widehat{\operatorname{tail}(s)}$. It follows that $\vdash_{\mathbf{L}} \xi \rightarrow \psi$, as $\xi$ is one of the disjuncts of $\psi$. So we get $\vdash_{\mathbf{L}} \xi \rightarrow \uplus_{I} \varphi$, and so $\uplus_{I} \varphi \in \operatorname{tail}(s)$ for tail $(s)$ is consistent.

Lemma 20 (truth). Given a signature $\sigma$, a formula $\varphi \in c l(\sigma)$, and a state $s$ of $\mathrm{M}^{\sigma}$,

$$
\mathrm{M}^{\sigma}, s \models \varphi \quad \text { iff } \quad \varphi \in \operatorname{tail}(s) .
$$

Proof. The proof is by induction on $\varphi$. The atomic and Boolean cases are easy to show. The cases for the modalities $\square_{i}(i \in \mathcal{I})$ and $\cap_{I}$ ( $I$ is an index) are not much different from those of the proof of Lemma 10 (we need to be careful with the closure, however; just note that all the $i$ 's and I's used here are bounded by an $\iota$ ). Here we detail the case for $\uplus_{I} \psi$.

$$
\begin{aligned}
& \mathrm{M}^{\sigma}, s \models \uplus_{I} \psi \\
\Leftrightarrow & \text { for all } t, \text { if }(s, t) \in \biguplus_{i \in I} \mathrm{R}_{i}^{\sigma} \text { then } \mathrm{M}^{\sigma}, t \models \psi \\
\Leftrightarrow & \text { for all } t, \text { if }(s, t) \in \biguplus_{i \in I} \mathrm{R}_{i}^{\sigma} \text { then } \psi \in \operatorname{tail}(t) \\
\Rightarrow & \uplus_{I} \psi \in \operatorname{tail}(s)
\end{aligned}
$$

For the converse direction of the last step, suppose $\uplus_{I} \psi \in \operatorname{tail}(s)$ and towards a contradiction that there is a state $t$ such that $(s, t) \in \biguplus_{i \in I} \mathrm{R}_{i}^{\sigma}$ and $\psi \notin \operatorname{tail}(t)$. So there is a path $\left\langle s_{0},\left\{i_{0}\right\}, \ldots,\left\{i_{n-1}\right\}, s_{n}\right\rangle$ of $\mathrm{M}^{\sigma}$ such that $\left\{i_{0}, \ldots, i_{n-1}\right\} \subseteq I$, $s=s_{0}$ and $t=s_{n}$.

- If $\mathrm{L}$ is $\mathrm{K}^{\cap \uplus}$ or $\mathrm{D}^{\bigcap \uplus}$, it must be that $t$ extends $s$ with $\left\langle J_{0}, \Phi_{1}, \ldots, J_{n-1}, \Phi_{n}\right\rangle$ where $\psi \notin \Phi_{n}$ and for each $x, i_{x} \in J_{x}$ and $\Phi_{x} \in$ MCS $^{\sigma}$. By definition tail $\left(s_{0}\right) \triangleright_{J_{0}} \Phi_{1} \triangleright_{J_{1}} \cdots \triangleright_{J_{n-1}} \Phi_{n}$. By the axioms $\uplus 1, \cap 1$ and $\cap 2$ we can get $\vdash_{\mathbf{L}} \uplus_{I} \psi \rightarrow \cap_{J_{0}} \uplus_{I} \psi$, and since $\cap_{J_{0}} \uplus_{I} \psi \in \operatorname{cl}(\sigma)$ we have $\uplus_{I} \psi \in \Phi_{1}$. Carrying this out recursively we get $\uplus_{I} \psi \in \Phi_{n}$ and so $\psi \in \Phi_{n}$ by $\mathrm{T} \uplus$, which contradicts $\psi \notin \operatorname{tail}(t)$.

- If $\mathrm{L}$ is $\mathrm{T}^{\cap \uplus}$, we face an extra case compared with the above, namely $s=t$. A contradiction can be achieved by applying the axiom $\mathrm{T} \uplus$.

- If $\mathrm{L}$ is $\mathrm{B}^{\cap \uplus}$, there are three cases: (i) $s_{x+1}=s_{x}$ or (ii) $s_{x}=\left\langle s_{x+1}, J, \Phi\right\rangle$ or (iii) $s_{x+1}=\left\langle s_{x}, J, \Phi\right\rangle$ where $J \supseteq I$ and $\Phi \in \mathrm{MCS}^{\sigma}$. In all cases, by similar 
reasoning to the above (for case (ii) we use the symmetric condition for $\triangleright_{I}$ ), we can show that $\psi \in \operatorname{tail}\left(s_{x+1}\right)$ given $\uplus_{I} \psi \in \operatorname{tail}\left(s_{x}\right)$, and then reach a contradiction similarly.

- If L is $\mathrm{S} 4^{\cap \uplus}, s_{x}(0 \leq x<n)$ must be an initial segment of $s_{x+1}$ and $s_{x+1} \backslash s_{x}$ is a finitary canonical $i_{x}$-path $\left(i_{x} \in I\right)$. By the axioms $\uplus 1$ and $\cap 1, \vdash_{\mathbf{S} 4} \mathbf{S}^{\cap \uplus}$ $\uplus_{I} \psi \rightarrow \cap_{\left\{i_{x}\right\}} \uplus_{I} \psi$. So we get $\uplus_{I} \psi \in \operatorname{tail}\left(s_{x+1}\right)$ (we use $\mathrm{T} \uplus$ in the case when $s=t)$. Recursively carrying this out, we get $\uplus_{I} \psi \in \operatorname{tail}(t)$, and so $\psi \in \operatorname{tail}(t)$ which leads to a contradiction.

- If L is $\mathrm{S} 5^{\cap \uplus}$, then $s_{x}$ and $s_{x+1}$ have a common initial segment $u$, and $s_{x} \backslash u$ and $s_{x+1} \backslash u$ are both finitary canonical $i_{x}$-paths. Since $\vdash_{\mathbf{S} 5} \cap \uplus \uplus_{I} \psi \rightarrow \cap_{\left\{i_{x}\right\}} \psi$ (for all $i_{x}$ ), $\cap_{I} \psi \in s_{0}$, and by the definition of $\triangleright_{I}, \cap_{I} \psi \in s_{x}$ for all $x$, so $\psi \in \operatorname{tail}(t)$ which leads to a contradiction as well.

Theorem 21 (weak completeness). Let $\boldsymbol{L}$ be the corresponding axiomatization introduced for a logic $L \in\left\{K^{\cap \uplus}, D^{\cap \uplus}, T^{\cap \uplus}, B^{\cap \uplus}, S 4^{\cap \uplus}, S 5^{\cap \uplus}\right\}$. For any $\varphi \in \mathcal{L}^{\cap \uplus}$, if $\models \varphi$, then $\vdash_{L} \varphi$.

Proof. Suppose $\nvdash_{\mathbf{L}} \varphi$. It follows that $\{\neg \varphi\}$ is $\mathbf{L}$ consistent. Extend it to be a maximal set $\Phi$ in $\operatorname{cl}((\mathbf{L}, \mathbf{L}, \neg \varphi, \iota))$ with $\iota$ the union of all the indices occurring in $\varphi$, then $\langle\Phi\rangle$ is a canonical path for $\mathbf{L}$ in $\operatorname{cl}((\mathbf{L}, \mathbf{L}, \neg \varphi, \iota))$. By the truth lemma, for any formula $\psi$, we have $\mathrm{M}^{(\mathrm{L}, \mathbf{L}, \neg \varphi, l)},\langle\Phi\rangle \models \varphi$ iff $\varphi \in \Phi$. It follows that $\Phi$ is satisfiable, which leads to $\not=\varphi$.

\section{Discussion}

We focused mainly on the completeness proof for the modal logics, K, D, T, B, S4 and S5, extended with intersection and with or without the transitive closure of union, but the method applies to many canonical multi-modal logics (including many of those normal modal logics between K and S5) with the intersection modality. By avoiding the model translation processes used in the unravelingfolding method and building a standard model directly, the proofs we present are dramatically simpler than those found in the literature. We believe that the readers who are familiar with the canonical model method for completeness proofs of modal logics will find the proofs very familiar and straightforward.

While our approach is inspired by simplifying the existing proof technique, the standard model we build is not identical to the model produced by the unraveling-folding processes: it is simpler because we do not have to use socalled reductions of paths. We emphasize, however, that the unraveling-folding method was still important for us to arrive at this proof technique: it explains why we take (finitary) canonical paths to be the states of the standard model. Further work that could be interesting is to show the bisimilarity of the model we build to that by the unraveling-folding processes.

We omit the details here, but our method can be applied directly to many other logics extended with intersection modalities, including popular systems of epistemic and doxastic logics such as S4.2, S4.3, S4.4 and KD45. 
Finally, it is worth mentioning that our proof technique is slightly more general than existing proofs in that it allows a (countably) infinite set of boxes. This slightly complicates the proofs in the cases with transitive closure of the union, requiring the use of the $\sigma$ signatures.

\section{References}

1. Ågotnes, T., Alechina, N.: Embedding coalition logic in the minimal normal multimodal logic with intersection. In: Ono, H., Ju, S. (eds.) Proceedings of the Second Asian Workshop on Philosophical Logic, Logic in Asia: Studia Logica Library, vol. 1. Springer International Publishing (2015)

2. Baader, F., Calvanese, D., McGuinness, D.L., Nardi, D., Patel-Schneider, P.F.: The Description Logic Handbook: Theory, Implementation, and Applications. Cambridge University Press, 2 edn. (2017)

3. Baader, F., Horrocks, I., Lutz, C., Sattler, U.: An Introduction to Description Logic. Cambridge University Press (2017)

4. Blackburn, P., de Rijke, M., Venema, Y.: Modal Logic, Cambridge Tracts in Theoretical Computer Science, vol. 53. Cambridge University Press, Cambridge University Press (2001)

5. Chellas, B.F.: Modal Logic: An Introduction. Cambridge University Press (1980)

6. Fagin, R., Halpern, J.Y., Vardi, M.Y.: What can machines know? on the properties of knowledge in distributed systems. J. ACM 39(2), 328-376 (1992)

7. Fagin, R., Halpern, J.Y., Moses, Y., Vardi, M.Y.: Reasoning about Knowledge. Cambridge, MA: The MIT Press (1995), hardcover edition

8. Halpern, J.Y., Moses, Y.: A guide to completeness and complexity for modal logics of knowledge and belief. Artificial Intelligence 54, 319-379 (1992)

9. Harel, D., Kozen, D., Tiuryn, J.: Dynamic Logic. The MIT Press (2000)

10. van der Hoek, W., Meyer, J.J.C.: Making some issues of implicit knowledge explicit. International Journal of Foundations of Computer Science 3(2), 193-224 (1992)

11. Meyer, J.J.C., van der Hoek, W.: Epistemic Logic for AI and Computer Science. Cambridge University Press, Cambridge, England (1995)

12. Wáng, Y.N.: Logical Dynamics of Group Knowledge and Subset Spaces. Ph.D. thesis, University of Bergen (2013)

13. Wáng, Y.N., Ågotnes, T.: Public announcement logic with distributed knowledge: Expressivity, completeness and complexity. Synthese 190(1 supplement), 135-162 (2013)

14. Wáng, Y.N., Ågotnes, T.: Relativized common knowledge for dynamic epistemic logic. Journal of Applied Logic 13(3), 370-393 (2015). https://doi.org/10.1016/j.jal.2015.06.004 